\title{
Widespread Traffic Congestion Prediction for Urban Road Network Based on Synergetic Theory
}

\author{
Liangliang ZHANG \\ Institute of System Engineering and Control School of Traffic and Transportation, Beijing Jiaotong \\ University, Beijing 100044, China \\ E-mail: 10114212@bjtu.edu.cn \\ Yuanhua JIA \\ Institute of System Engineering and Control School of Traffic and Transportation, Beijing Jiaotong \\ University, Beijing 100044, China \\ E-mail: yhjia@bjtu.edu.cn \\ Zhonghai NIU \\ Institute of System Engineering and Control School of Traffic and Transportation, Beijing Jiaotong \\ University, Beijing 100044, China \\ Cheng LIAO \\ Institute of System Engineering and Control School of Traffic and Transportation, Beijing Jiaotong \\ University, Beijing 100044, China

\begin{abstract}
The traffic congestion often occurs in urban road network. When one of the sections becomes congested, it will lead to a series of congestions in other sections. The traffic congestion spreads rapidly until part of road network becomes congestion ultimately. In this case, the paper investigates the mechanism of the traffic congestion in urban road network and points out that subsystems of the traffic congestion always perform completive and cooperative functions in the process of traffic congestion. The process behaves in a manner of self-organized criticality, which can be forecasted. The paper also establishes synergetic predictive models based on self-organized criticality of the synergetic theory. Finally, the paper takes Beijing road network as an example to forecast the widespread traffic congestion. The result shows that the established models are accuracy, and the traffic congestion is featured of self-organized criticality.
\end{abstract}

Keywords urban road network; traffic congestion; self-organized criticality; synergetic theory; predictive model

\section{Introduction}

With the development of economy-society and increasing of population in urban area, the motor vehicle possessions and the vehicle demands for the road transportation enjoy rapidly growth. The traffic congestion has become a common problem in many metropolises of worldwide. Some Chinese cities, for example, Beijing, Shanghai, Guangzhou and etc., have terrible

Received November 27, 2013, accepted January 21, 2014

Supported by the National Natural Science Fund Committee (71340020) 
traffic congestion in every section and every day. It brings frequent traffic accidents, low working efficiency of the road network and even seriously impact on the urban economy development. Traffic congestion prediction plays an important role in intelligent traffic control system to allow the traffic management department to do better management. Real-time and reliable prediction on traffic congestion could provide the support for the decision-making on traffic management. With the traffic congestion getting worse, experts and scholars have paid attention on the civil work engineering as well as the information technology. Early researches focused on prediction based on one-dimensional traffic time series, such as [1] and [2]. Recently, the trend has been shifted to prediction based on spatial temporal correlations of traffic flows, such as [3] and [4]. For instance, the vector ARMA model, such as [5], incorporating both spatial and temporal correlations, such as [6]; the spatial econometrics models focus on congestion propagation over adjacent links, such as [7]; and others focused on feature selection for traffic congestion prediction, such as [8]. Those results have promoted the development of traffic congestion theory. Macroscopically, the urban road network is an open system which exchanges energy and traffic flow with the outside constantly. The traffic jam in the urban road network occurs originally at a certain part of network and gradually expands to the other parts. Finally, it will result in congestion of the entire network. In this process, the sections of the road network show a cooperative and systematic efficiency and the evolution of nonlinear systems is followed. In terms of traffic congestion, it experiences three stages: equilibrium state, near equilibrium state and far from equilibrium state. Therefore, this paper uses the basic principles and achievements of synergetic theory to research the traffic congestion: if the congestion occurs in a part of a work. It will be extended to the entire network. The paper also establishes the traffic congestion evolution model to forecast the time of the entire congestion and the conclusion could provide support to the traffic management sector.

\section{Basic principle of synergetic theory}

The synergetic principle is put forward by Hennenn Haken, a German professor. It studies how the system that is far from equilibrium state generates time, space or functional structure through the self-organization among various subsystems. Its main goal is to find a universal law among the vastly different phenomena in the real world. Synergetic theory believes that the world's unity lies not only in the singleness of their microscopic structure, but also in the formation of macroscopic structures complying with certain universal laws. Haken described the behavior near the critical point in synergetic theory and proposed server principle and order parameter principle, which considered that the evolution is controlled by the order parameter and the final structure of evolution and the degree of ordering depends on the order parameter. In synergetic theory, the critical behaviors of all the amount of the subsystem status and the coupling between them are divided into two variables. Fast relaxing variables (fast variables). Fast relaxing variables damp at large decay in critical. They do not have a big influence on the nature of the evolution of the system. There are tens of thousands of variables in a system, but the vast majority critical behavior of state variables are such fast relaxing variables; Slow relaxing variables (slow variables). The behavior in the critical of these kinds of slow relaxing variables have no significant difference with fast relaxing variables, but they show critically 
undamped phenomena when the system reaches a critical point. The number of such variables is minimal in a system, but they drive the movement of other fast relaxing variables and decide the final status and structures of the system evolution. In Synergetic theory, the order parameter is the characterization of the nature and extent of the system after an orderly phasechange. Under the old structure before the phase transition, the order parameter is 0 , and then the order parameter is nonzero from the phase transition point. Order parameter has three characteristics. Synergetic theory studies the macroscopic behavior of the system consisting of a large number of components. The introduced order parameter is the macro parameter and it is used to describe the overall behavior of the system. Order parameter is the product of collective micro-subsystem movements as well as the behavior and judgment of cooperative effects. Order parameter controls the subsystem behavior and dominates the evolution of system.

\section{Order parameter evolution equation of traffic congestion}

According to synergetic theory, the overall behavior of the system is controlled by the order parameter when the system is near the critical point. The final structure and the degree of ordering of evolution depend on the order parameter. The size of the order parameter can be used to mark the extent of macroscopic order. When the system is disordered, the order parameter is zero. As the external conditions change, the order parameters also change. The order parameters grow to the maximum at the critical point. In this case, an orderly organized macro structures appears. As to traffic jams forming process, its order parameter can be taken to determine the increasing number of the system's traffic section. This is because it fulfills the order parameter characteristics while the order parameter is the slow variables. Simply, the evolution of the traffic jams could be explained only by two kinds of variables $(\mu, s)$. Accordingly, we determine the system evolution equations. Generally, it uses the form of Langevin equation.

$$
\dot{\mu}=K(\mu, s)+F(t)
$$

Where, $K(\mu, s)$ is a nonlinear function which contains the fast variables and slow variables; $\mu$ represents slow variables, $s$ represents fast variables; $F(t)$ means fluctuating force. Equation (1) indicates that the evolution of any non-linear system is not only controlled by the internal factors (nonlinear interaction among the various subsystems), which can be shown by a nonlinear

function $K(\mu, s)$. It is also influenced by external factors, which can be represented by a fluctuation function $F(t)$. Internal factors are the nature reasons for the system evolution while the mainly role of external factors is to promote internal factors to changes and to trigger the qualitative in critical point. Since the external factors are not the dominated factors for traffic congestion. To simplify the model, we will not consider the impact of external factors, namely ignorance of the fluctuation function in equation (1). For the two-dimensional system, the nonlinear function in formula (1) can be expressed as follow:

$$
k(\mu, s)=\alpha \mu-\mu s
$$

Based on the equation (1) and equation (2), we can conclude that

$$
\dot{\mu}=\alpha \mu-\mu s
$$


Where $s$ can generally be expressed as follows

$$
\dot{s}=-\beta s+\mu^{2}
$$

In equation (3) and (4), the $s$ can be expressed by $\mu$. Then, we get the equation (5).

$$
s(t)=\int_{-\infty}^{+\infty} \mathrm{e}^{-\beta(t-\tau)} \mu^{2}(\tau) \mathrm{d} \tau
$$

The $s(t)$ in equation (5) can be converted into $\mu(t)$ by using distribution integration method, i.e.

$$
s(t)=\mu^{2} / \beta-\int_{-\infty}^{+\infty} \mathrm{e}^{-\beta(t-\tau)} 2(\mu \dot{\mu}) \mathrm{d} \tau / \beta
$$

When $\mu$ becomes slower, $\dot{\mu}$ become small. In this case, if we ignore the integral term in equation (6), we can get equation

$$
s(t) \approx \mu^{2}(t) / \beta
$$

Put the equation (7) into equation (3) to get

$$
\dot{\mu}=a \mu-b \mu^{2}
$$

Where $b$ is $1 / \beta$. Equation (8) shows that fast variables can be shown by slow variables. That means the fast variables change their behavior when the slow variables do and they are server to the slow variables. As in the formula from (3) to (8) using slow variables to describe fast variable, it is the well-known adiabatic elimination. It should be noted that: the collaborative adiabatic elimination is a phased approach to the process evolution of fast variables and the slow variables in the critical state, so it has a strong applicability in phases. It is a very effective method when the evolution is in critical point.

\section{Collaborative prediction for urban road network}

In equation (8), the paper replaces the slow variables $u$ with the number of the traffic jams points and results in time series data of the congestion point in urban road network. Then the paper uses least squares fitting and gets the results of variables $a$ and $b$ according to equation (8). Then, we can forecast the time of the widespread traffic congestion. We ignore the fluctuation function $F(t)$ in the process of getting equation (8). At the same time, the traffic congestion differs in different situation. In order to reduce forecasting errors and increase the prediction accuracy, the paper uses cumulative sum method to calculate the original data. The original time series is $\mu_{0}$, and the first order time series is $\mu_{0}$.

$$
\left\{\begin{array}{l}
\mu_{0}=\left[\mu_{0}(0), \mu_{0}(1), \cdots, \mu_{0}(n)\right] \\
\mu_{1}=\left[\mu_{1}(0), \mu_{1}(1), \cdots, \mu_{1}(n)\right]
\end{array}\right.
$$

The first order CUSUM is equation (10):

$$
\mu_{1}\left(t_{i}\right)=\mu_{1}\left(t_{i-1}\right)+\mu_{0}\left(t_{i}\right), \quad i=1,2, \cdots, n
$$

Likewise, according to adiabatic elimination, the equation (8) is defined as equation (11).

$$
\mathrm{d} \mu_{1}(t) / \mathrm{d} t=a \mu_{1}-b \mu_{1}^{2}
$$


Then, the paper solves differential equations (11), and the result is shown in equations(12).

$$
\mu_{1}(t, a, b)=\sqrt{a /\left(1 /\left[\frac{\mu_{1}(0)}{a-b \mu_{1}^{2}}\right] \mathrm{e}^{2 a t}+b\right)}
$$

Where $\mu_{1}(0)$ is the number of increasing traffic performance index at the start time; variables $a$ and $b$ are undetermined coefficient. Equation (11) reflects the process of the congestion evolution and the right term of the equation (11) is actually growth rate of traffic performance index. Therefore, we can take the greatest number of congestion points as widespread traffic congestion prediction time. So we get equation (13) from equation (12).

$$
Y\left(\mu_{1}\right)=a \mu_{1}-b \mu_{1}^{2}
$$

We differentiate the equation (13). Then $Y\left(\mu_{1}\right)$ has a max value when $\mu_{1}$ value is $\sqrt{a / 3 b}$. According to equation (12), we get the traffic congestion time as follows:

$$
t=\left\{\ln \left[a-b \mu_{1}^{2}(0)\right] / 2 b \mu_{1}(0)\right\} / 2 a
$$

\section{Case analysis}

The paper takes widespread traffic congestion prediction of Beijing urban road network as an example. Because there is no uniform standard about what is widespread traffic congestion, the paper uses the standards which are measured by Beijing Transportation Research Center, called Traffic Performance Index (TPI). The value scope of traffic performance index is from 0 to 10, and it graduates into five levels. Then the paper gets the traffic congestion standards in Table 1.

Table 1 The group standards of traffic states

\begin{tabular}{lll}
\hline TPI & Traffic states & Road network condition \\
\hline$[0,2)$ & Unblocked traffic & Road network condition is very well, and traffic congestion does not occur. \\
{$[2,4)$} & Little traffic & Road network condition is well, and there is a small amount of traffic \\
& congestion & congestion. \\
{$[4,6)$} & Mild congestion & Road network condition is poor, and parts of road becomes congested. \\
{$[6,8)$} & Moderate & Road network condition is very poor, and a large number of road become \\
& congestion & congested. \\
{$[8,10]$} & Serious congestion & The traffic congestion is very serious, and almost roads become congested. \\
\hline
\end{tabular}

In order to validate the proposed method, the paper chooses the Traffic Performance Index which is published in the internet, and calculates the increasing value before $15 \mathrm{~min}$.

Table 2 The increasing value of traffic performance index

\begin{tabular}{cccccc}
\hline $6: 30$ & $6: 45$ & $7: 00$ & $7: 15$ & $7: 30$ & $7: 45$ \\
\hline 0.1 & 0.7 & 1.3 & 1.5 & 2.1 & 1.8 \\
\hline
\end{tabular}

According to equation (11), we use the data from Table 2 to get the results of $a=0.6718$, $b=0.0093$. Then, we get the time of the large congestion time from equation (14). The result of $t$ is 6.10 , and the real time is 8:02. In fact, the time of widespread traffic jams is from 7:50 to 8:00, and the maximum error of forecasting is 12 minutes. From the result, we could consider that the theory and model could be used for the congestion forecasting. 


\section{Conclusion}

Based on the above case analysis and research, synergetic theory could reflect the process of the network congestion very well. The established model could be used to forecast the congestion by using limited data we have before the widespread of traffic congestion. The established model is only applicable to forecast the critical moment of traffic network congestion. It should be noted that this paper is a preliminary research and its universality still needs to be further studied.

Acknowledgements We thank the referees for their time and comments.

\section{References}

[1] Smith B L, Williams B M, Oswalsd R K. Comparison of parametric and nonparametric models for traffic flow forecasting. Transportation Research Part C, 2002, 10: 303-321.

[2] Williams B M, Hoel L A. Modeling and forecasting vehicular traffic flow as a seasonal ARIMA process: Theoretical basis and empirical results. Journal of Transportation Engineering - ASCE, 2003, 129: 664672.

[3] Ben-Akiva M E, Gao S, Wei Z, et al. A dynamic traffic assignment model for highly congested urban networks. Transportation Research Part C, 2012, 24: 62-82.

[4] He J, He Q, Swirszcz G, et al. Ensemble-based method for task 2: Predicting traffic jam. Proc. IEEE International Conference on Data Mining Workshops, 2010: 1363-1365.

[5] Chandra S R, Al-Deek H. Predictions of freeway traffic speeds and volumes using vector autoregressive models. Journal of Intelligent Transportation Systems, 2009, 13(2): 53-72.

[6] Min W, Wynter L. Real-time road traffic prediction with spatio-temporal correlations. Transportation Research Part C, 2011, 19: 606-616.

[7] Hu J, Kaparias I, Bell M G H. Spatial econometrics models for congestion prediction with in-vehicle route guidance. IET Intelligent Transport Transportation System, 2009, 10: 246-254.

[8] Su Yang. On feature selection for traffic congestion prediction. Transportation Research Part C, 2013, 26: 160-169. 